\title{
Kardiovaskuläre Risiken nach Androgen-Entzug
}

Hintergrund und Fragestellung: Der Androgen-Entzug (AE) ist die erste systemische Behandlungslinie beim disseminierten Prostatakarzinom (PCa). Abgesehen von seinem therapeutischen Nutzen erhöht der AE das Risiko verschiedener metabolischer Störungen: Abnehmende Insulin-Sensitivität, Veränderungen des Lipid-Profils verbunden mit einem wachsenden $\mathrm{Ri}$ siko kardiovaskulärer Erkrankungen (CVD). Ziel war es, die Assoziation zwischen dem CVD-Risiko und der Dauer und Art des AE bei Männern mit Prostatakarzinom zu klären.

Patienten und Methoden: Basierend auf den Daten des Nationalen Schwedischen Gesundheitsregisters, wurde das Risiko einer CVD in einer Kohorte von

Originalie

O'Farrell et al. Risk and timing of cardiovascular disease after androgen-deprivation therapy in men with prostate cancer.

J Clin Oncol. 2015;33(11):1243-51. 41.362 Männern mit PC unter AE analysiert und mit einer altersgleichen Kohorte ohne PC $(n=187.785)$ verglichen.

Ergebnisse: Von 2006 bis 2012 waren 10.656 Männer mit Antiandrogenen (AA), $26.959 \mathrm{mit}$
GnRH(Gonadotropin-Releasing-Hormon)-Agonisten behandelt worden und 3.747 orchiektomiert. Gegenüber der Kontrollgruppe war die CVD-Rate erhöht bei Patienten unter der Therapie mit GnRH-Agonisten (Hazard Ratio [HR] zur Inzidenz von CVD 1,21; 95\%-Konfidenzintervall [95\%-KI] 1,18-1,25) und Orchiektomie (HR 1,16; 95\%-KI 1,08-1,25). Männer mit PC unter AA hatten ein reduziertes CVD-Risiko (HR 0,87; 95\%-KI 0,82-0,91). Das CVDRisiko war am höchsten in den ersten 6 Monaten des AE bei Männern, die schon vor der Therapie zwei oder mehr kardiovaskuläre Ereignisse erfahren hatten: Bezogen auf die Vergleichskohorte betrugen während der ersten 6 Monate die HRs für CVD unter GnRH-Agonisten 1,91 (95\%-KI 1,66-2,20), unter AA 1,60 (95\%-KI $1,24-2,06)$ und nach Orchiektomie 1,79 (95\%-KI 1,16-2,76).

Schlussfolgerung der Autoren: Die Indikation zum Androgenentzug sollte bei Männern mit PC streng gestellt werden, damit der Nutzen einen möglichen Schaden überwiegt - insbesondere bei Männern mit kurz zurückliegender Anamnese einer CVD.

Schlüsselwörter: Prostatakarzinom - antiandrogene Therapie - Androgenentzug - kardiovaskuläre Risiken - Bewegung

\section{- Kommentar von Ulrich R. Kleeberg, Hamburg}

\section{„AE scheint kardioprotektiven Effekt des Testosterons zu reduzieren"}

Das erhöhte Risiko kardiovaskulärer Erkrankungen für Männer mit PC unter AE ist seit Jahren bekannt. Verschiedene Ursachen legen nahe, dass die AE mit dem kardioprotektiven Effekt des Testosterons interferiert [1]. Auch könnte das relative oder absolute Überwiegen des endogenen oder exogenen Östrogens nach oraler Östrogentherapie hierfür verantwortlich sein [2]. $\mathrm{GnRH}$-Agonisten aktivieren zudem zirkulierende $\mathrm{GnRH}$-Rezeptor-exprimierende T-Zellen, die arteriosklerotische Plaques destabilisieren und desintegrieren [3]. Interessant ist, dass das CVD-Risiko nach einer Orchiektomie vergleichsweise hoch, dagegen unter $A A$, die den Testosteron-Spiegel nicht reduzieren, niedrig ist. Eine Verminderung des Testosteron-Spiegels bedingt einen Anstieg des Low-Density-Lipoproteins, der Triglyzeride und des Insulins; alles drei Komponenten des metabolischen Syndroms, ein starker Risikofaktor für CVD [4].

Für Onkologen ist es grundsätzlich entscheidend, besonders aber bei chronologisch wie biologisch „älteren“ Männern, vor Aufnahme einer AA-Therapie, eine sorgsame Eigen- wie Familienanamnese und ein (geriatrisches) Assessment mit klinischer Untersuchung durchzuführen. Bei Risikofaktoren wie metabolischem Syndrom (Diabetes mellitus, Hypertonie, Adipositas), kardiovaskulären Vorerkrankungen und/oder ungesunder Lebensführung (Genussmittel, Bewegungsarmut) gilt es, die Indikation zu einer systemischen endokrinen Therapie besonders streng zu stellen. Lassen die Prognosefaktoren des Prostatakarzinoms eine langsame Progredienz ohne Komplikationen erwarten, kann Abwarten mit kompetenter Verlaufsbeobachtung
- quo ad vitam - von entscheidendem Vorteil sein, ein unbedachtes Vorgehen dagegen iatrogene Komplikationen induzieren. Ist eine therapeutische Intervention unabdingbar, sollten, wie von O'Farrell und Kollegen vorgeschlagen, zuerst AA (Flutamid, Bicalutamid) eingesetzt werden, da sie den TestosteronSerumspiegel nicht beeinträchtigen. In jedem Falle sind die Patienten bei isokalorischer, ggf. gewichtsmindernder Kost zu täglicher Bewegung anzuhalten (eine Stunde flottes Gehen, Radeln, Schwimmen, etc.) - immer und immer wieder, um die Adherence zu sichern. Nachsorge wird so zur Vorsorge.
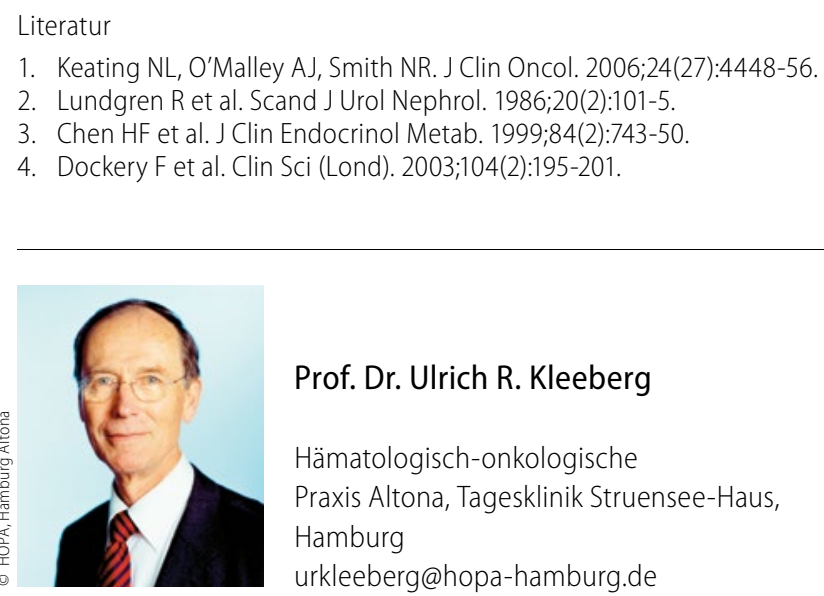

Prof. Dr. Ulrich R. Kleeberg

Hämatologisch-onkologische

Praxis Altona, Tagesklinik Struensee-Haus,

Hamburg

urkleeberg@hopa-hamburg.de 